\title{
Article
}

\section{Decomposing Dual Scale Soil Surface Roughness for Microwave Remote Sensing Applications}

\author{
Philip Marzahn ^, Moritz Seidel and Ralf Ludwig \\ Department of Geography, Ludwig-Maximilians University, Luisenstrasse 37, D-80333 Munich, \\ Germany; E-Mails: moritzseidel@gmx.de (M.S.); r.ludwig@lmu.de (R.L.) \\ * Author to whom correspondence should be addressed; \\ E-Mail: p.marzahn@iggf.geo.uni-muenchen.de; Tel.: +49-89-2180-6698; Fax: +49-89-2180-6675.
}

Received: 15 May 2012; in revised form: 28 June 2012 / Accepted: 30 June 2012 /

Published: 6 July 2012

\begin{abstract}
Soil surface roughness, as investigated in this study, is decomposed in a dual scale process. Therefore, we investigated photogrammetrically acquired roughness information over different agricultural fields in the size of 6-22 $\mathrm{m}^{2}$ and decomposed them into a dual scale process by using geostatistical techniques. For the characterization of soil surface roughness, we calculated two different roughness indices (the RMS height $s$ and the autocorrelation length $l$ ) differing significantly for each scale. While we could relate the small scale roughness pattern clearly to the seedbed rows, the larger second scale pattern could be related to the appearance of wheel tracks of the tillage machine used. As a result, major progress was made in the understanding of the different scales in soil surface roughness characterization and its quantification possibilities.
\end{abstract}

Keywords: soil surface roughness; photogrammetry; SAR; synthetic aperture radar; detrending; RMS height; autocorrelation

\section{Introduction}

Soil surface roughness has a strong impact on the backscattered signal of an imaged surface by a microwave signal. For the parametrization of available backscatter models (e.g., IEM, Oh's model), the illuminated soil surface is strongly generalized and assumed to be a single scale stationary isotropic process [1]. However, recent studies have shown a strong non-isotropic behaviour of soil surfaces 
under agricultural use [2,3]. Thus, the inadequate representation of soil surface roughness leads to insufficient results in the derivation of geophysical variables, e.g. soil moisture. In addition, recent studies have shown a huge impact of soil surface roughness and its orientation on the backscattered signal under slightly different look directions of the sensor [4]. This causes a backscatter difference of several $\mathrm{dB}$ due to the anisotropic appearance of the soil surface in agricultural landscapes. In several theoretical studies, Ulaby et al. [5] as well as Shin et al. [6] investigated the scattering from anisotropic quasi-periodic surfaces and described scattering with three coherent and incoherent terms originating from an anisotropic roughness component, a combined isotropic and anisotropic component and a deterministic periodical function. Using this model one can predict the anisotropic dependency of the scattering for row orientations with a width of $90^{\circ}$. On the ground truth site, Marzahn et al. [7] showed a significant difference in roughness values originating from the orientation of roughness measurements to the seedbed rows. Mattia [8] developed a backscatter model based on the findings of [6] which is able to reproduce the very narrow directional scatter pattern observed in [4] of only a few tenths of a degree. From the findings of [6] and especially of [4,8], the periodical roughness component has a strong impact on the backscattered signal. Besides the anisotropic appearance, different scales of soil surface roughness have an impact on the backscattered signal. Roemkens et al. [9] defined different scale dependent roughness classes in the context of a landscape (see Table 1). However, there exist some disparities in the classification scheme, for example a typical grain crop (wheat, barley) shows a row distance of 5-15 cm, thus the borders of the class orientated roughness are ambiguous.

Table 1. Roughness classification after [9].

\begin{tabular}{lll}
\hline Roughness Class & Scale $[\mathrm{mm}]$ & Topographic Elements \\
\hline micro roughness & $\leq 2$ & texture dependent roughness \\
random roughness & $2-200$ & soil aggregates and clods \\
orientated roughness & $200-400$ & rows, tillage patterns \\
higher order roughness & $\geq 400$ & slope, field borders \\
\hline
\end{tabular}

In the context of microwave remote sensing, only the random and orientated roughness components as well as the higher order roughness class have a direct impact on the backscattered signal. However the latter can be reliably corrected by applying a terrain correction comprising a digital elevation model. In the available backscatter models, the random and orientated roughness components are usually treated as a single scale process, simplifying the proposed definition of [9]. Thus, available roughness measurements and their characterization of roughness patterns are mostly carried out by using a laser profiler, or mesh board, providing a generalized single scale roughness description [10-12]. Indeed, state of the art descriptions of soil surface roughness patterns included the measurements of soil surface roughness by photogrammetric acquisition systems (e.g., [2,13-16]. Especially Aguilar et al. [14] highlighted the advantage of using a photogrammetric acquisition system instead of a laser device due to the in-field acquisition speed. In their study, Blaes et al. [2] first described a dual scale approach for the characterization of soil surface roughness in an agricultural environment. At sizes of $3.4 \times 2.4 \mathrm{~m}^{2}$, they characterized for a single sample plot two different roughness scales which they related to the seedbed rows and, as a superimposition, the random appearance of soil clods. However, they only defined these 
two scales for the autocorrelation length $l$ differing only in a range of $10-20 \mathrm{~cm}$, while for another roughness index, common in microwave remote sensing, the RMS-height $s$ is not considered. Zribi et al . [16] characterized the multi-scale appearance of an agricultural soil surface by a fractal model and for the large scale roughness component with the RMS height and the autocorrelation length respectively autocorrelation function. They evaluated the impact of these three roughness scale dependent terms on the backscattering and concluded a better understanding of the backscattering by incorporating such terms and scales in future backscatter models. In a theoretical experiment Shin et al. [6] and Mattia [8] developed a scatter model comprising a two scale roughness description.

The findings of [16] as well as [2] were evaluated on samples of $1 \times 1 \mathrm{~m}^{2}$, respectively $3.4 \times 2.4 \mathrm{~m}^{2}$. However, the size of the sample plot determines the ability to characterize soil surface roughness in an agricultural environment. As shown by several authors [17,18], the length (size) of a sampling area has a strong impact on the retrieved roughness indices. Thus, with an increase in the sampling size, one can observe a significant change in the estimated roughness indices. To characterize the periodicity of an agricultural soil surface, it is necessary to acquire sample plots larger than the proposed size by [2] in order to describe a statistically robust roughness index for the different scales of soil surface roughness.

In this paper, we will present a method to characterize soil surface roughness using high resolution, photogrammetrically acquired digital surface models (DSM). As the periodical (horizontal) roughness component has a significant impact on the backscattered signal [4], we will analyze the periodical roughness component and propose a method to decompose its multi-scale appearance in its single contributions. We first describe the generation of the DSMs, which provides the basis for the calculation of two different roughness indices. In an extensive analysis, the proposed method for the decomposition of the different roughness scales is described and the effect of detrending the acquired DSMs on the retrieval of the roughness indices is highlighted. Section 3 summarizes the results of the proposed methods in the context of microwave remote sensing applications.

\section{Methods}

\subsection{Roughness Acquisition}

The setup for acquisition of soil surface roughness information consists of a customized Canon EOS 5D used with a Canon EF 2/35 mm lens and a reference frame (see Figure 1). Usually, metric cameras are used for photogrammetric image acquisitions. However, recent studies [19] have shown the usability of non-metric cameras for photogrammetric applications at reduced costs. Indeed, the geometric stability of these cameras is mostly the limiting factor in terms of achievable accuracy $[20,21]$ and thus altering the interior orientation. To increase the stability of the camera, one can mechanically stabilize the lens by using glue or fix the sensor to the camera's body using screws [22]. The off-the-shelf Canon EOS 5D in combination with the Canon EF $35 \mathrm{~mm}$ lens produces a maximum absolute Length Measurement Error (LME) of $330 \mu \mathrm{m}$, without fixation of the focusing tube at a maximum object distance of 2.5 $\mathrm{m}$. Fixing the lens by placing epoxy resin between the focusing tube and the outer lens tube reduces the maximum absolute LME to $47 \mu m$ [19]. After fixation of the lens, the camera was calibrated and its interior orientation estimated by Aicon 3D Studio in conjunction with an external three dimensional calibration test field [19]. Table 2 shows the results of the calibration process. 
Figure 1. Reference frame for relative orientation of the images acquired for DSM generation and detailed view of a GCP.

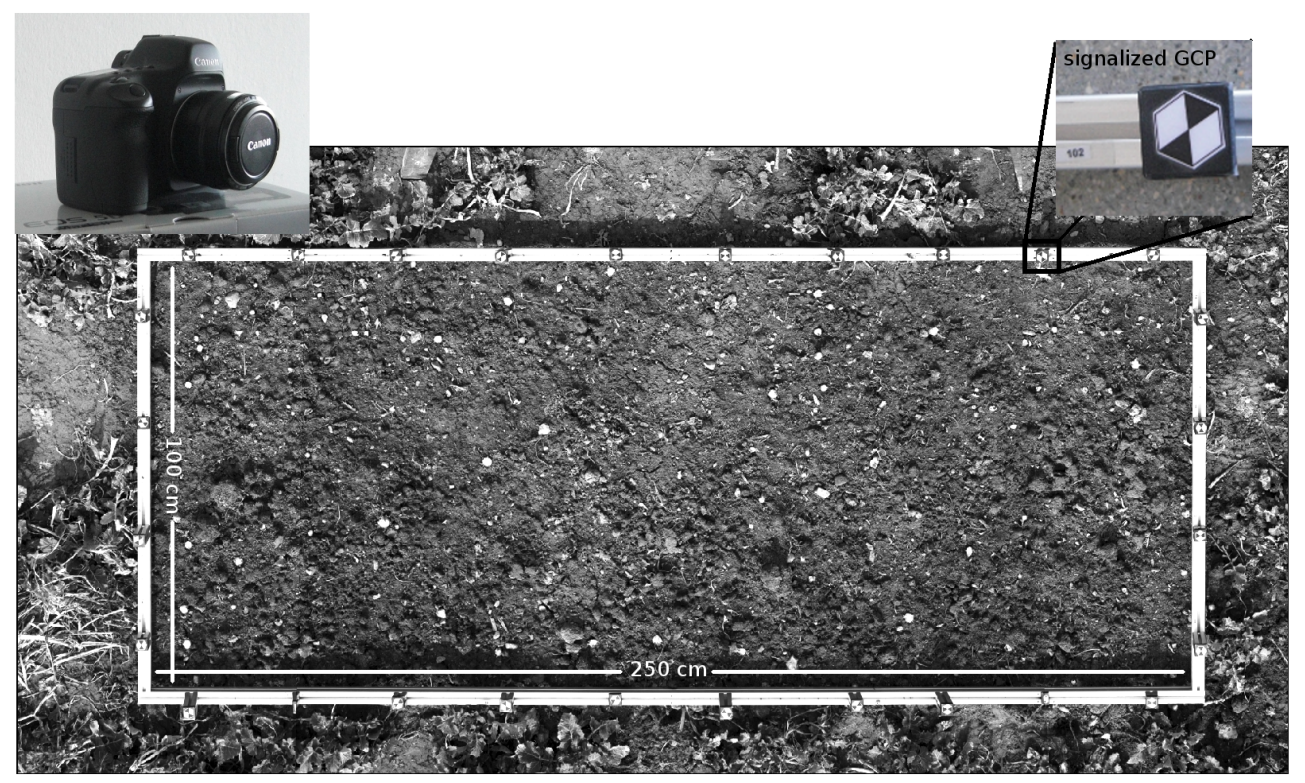

Table 2. Canon EOS 5D characteristics and calibration results. Calibration with Aicon 3D Studio [23] — parameters converted for input in LPS, K1 = 3rd-order term of radial distortion correction, $\mathrm{K} 2=5$ th-order term of radial distortion correction, $\mathrm{K} 3=7$ th-order term of radial distortion correction, $\mathrm{P} 1$ and $\mathrm{P} 2$ = Coefficient of decentering distortion.

\begin{tabular}{lll}
\hline Parameter & Values & Standard Error \\
\hline Camera & Canon EOS 5D & - \\
Lens & Canon EF 2/35 mm, fixed focus at & - \\
& $2.5 \mathrm{~m}$ & \\
Resolution & $4,368 \times 2,912$ pixels & - \\
Pixel width $x y[\mathrm{~mm}]$ & 0.0082 & - \\
Focal length $c[\mathrm{~mm}]$ & 35.8919 & 0.0000 \\
Principal point offset $x p[\mathrm{~mm}]$ & -0.2026 & 0.0000 \\
Principal point offset $y p[\mathrm{~mm}]$ & 0.2092 & 0.0000 \\
K1 $[\mathrm{mm}]$ & $7.30312 \mathrm{e}-005$ & $8.2836 \mathrm{e}-008$ \\
K2 $[\mathrm{mm}]$ & $-5.30725 \mathrm{e}-008$ & $5.535 \mathrm{e} 010$ \\
K3 $[\mathrm{mm}]$ & $-2.33223 \mathrm{e}-011$ & $1.1034 \mathrm{e}-012$ \\
P1 $[\mathrm{mm}]$ & $3.2677 \mathrm{e}-005$ & $1.296 \mathrm{e}-007$ \\
P2 $[\mathrm{mm}]$ & $-1.3050 \mathrm{e}-005$ & $1.167 \mathrm{e}-007$ \\
\hline
\end{tabular}

To numerically characterize soil surface roughness, DSMs generated out of the acquired imagery are the basis for further analyses. The generation of the DSMs was done using Leica Photogrammetry Suite (LPS). As LPS needs to establish exterior orientation for DSM generation, known ground control points (GCPs) with xyz coordinates are mandatory. Therefore a reference frame measuring $1 \times 2.5 \mathrm{~m}^{2}$ was set-up, providing 28 horizontally and vertically distributed GCPs. The co-ordinates of the GCPs were determined using a calliper ruler with an accuracy of $0.1 \mathrm{~mm}$ in the $\mathrm{z}$ direction. Six images acquired from a height of $2.5 \mathrm{~m}$ are necessary to cover the whole frame. Table 3 summarizes the characteristics of 
the image acquisition. As the frame is limited in size to $1 \times 2.5 \mathrm{~m}^{2}$, it is necessary, for larger roughness acquisitions, to acquire consecutive image acquisitions of the frame by moving the frame along a leveled plane which is ensured by an align fixture (see Figure 2).

Table 3. Flight plan for the coverage of the reference frame.

\begin{tabular}{ll}
\hline Parameter & Values \\
\hline Sensor altitude $h[\mathrm{~mm}]$ & 2,500 \\
Base length $b[\mathrm{~mm}]$ & 1,000 \\
$h / b$ & 2.5 \\
Image scale & $1: 69.65$ \\
Ground sample distance $[\mathrm{mm}]$ & 0.57 \\
Forlap x [\%] & 50 \\
Sidelap y [\%] & 60 \\
Number of images per strip & 3 \\
Number of strips & 2 \\
\hline
\end{tabular}

Figure 2. Roughness acquisition scheme and image arrangement for stereo coverage of the frame.

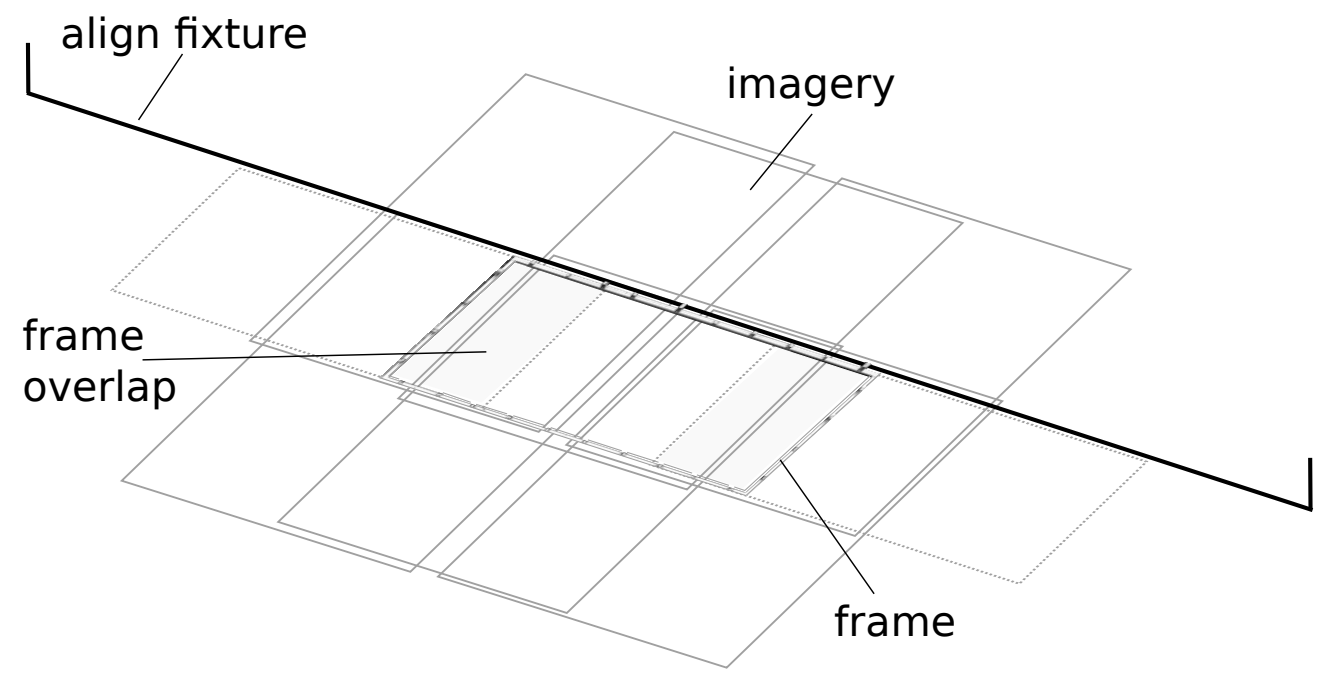

From the acquired imagery, DSMs were generated using LPS. As LPS uses epipolar constraints during image matching based on the established exterior orientation of the imagery, the exterior orientation of the block first has to be established. We therefore used the GCPs installed on the frame and improved the original camera calibration by using the Ebner model [24] which is implemented in LPS. After the exterior orientation has been established, the DSMs were generated using different matching strategies depending on the differences in elevation. Table 4 summarizes the applied strategies based on a ground pixel size of $0.57 \mathrm{~mm}$. For rougher surfaces a larger search window is chosen. Indeed, the surfaces showed similar smooth roughness classes without any significant change in roughness, therefore the applied strategies vary only little. Using an epipolar based approach, Linder [25] suggests a correlation coefficient threshold for the matching process for such applications of 0.65 . In a laboratory experiment, 
Heng et al. [26] as well as Rieke-Zapp et al. [22] used a correlation coefficient of 0.80. In our study, under field conditions, the correlation coefficients for matching two pixels showed good results at 0.75 . After successful generation of DSMs, a $7 \times 7$ pixel low pass filter was applied to remove peaks to smoothen the DSMs and resampled to a regular resolution of $2 \times 2 \mathrm{~mm}^{2}$.

Table 4. Matching strategies used during DSM generation as required by LPS based on a pixel size in ground space of $0.57 \mathrm{~mm}$ in the $\mathrm{x}$, $\mathrm{y}$ direction.

\begin{tabular}{llll}
\hline Roughness Class & $\begin{array}{l}\text { Search Window } \\
{[\text { pixel }]}\end{array}$ & $\begin{array}{l}\text { Correlation } \\
\text { Window }[\text { pixel }]\end{array}$ & $\begin{array}{l}\text { Correlation } \\
\text { Coefficient Threshold }\end{array}$ \\
\hline seedbed & $25 \times 3$ & $11 \times 11$ & 0.75 \\
crusted seedbed & $21 \times 3$ & $11 \times 11$ & 0.75 \\
smooth crusted & $17 \times 3$ & $11 \times 11$ & 0.75 \\
\hline
\end{tabular}

Roughness Samples

Several field campaigns for the measurement of soil surface roughness were scheduled in May 2011 over the Wallerfing test site, which is part of the SMO Cal/Val activities [27] located in the Upper Danube watershed approximately $100 \mathrm{~km}$ northeast of Munich. The region, which has a low relief energy, is mainly agricultural in character, soils mainly consist of loess loam and the main crops are winter wheat, winter barley, corn and sugar beet. During the campaign most of the crops had been already sown several weeks ago and were already at the beginning of their growth. The seedbed structure was at all sample points still well developed, however the random occurrence of soil clods was limited due to precipitation (see Figure 3). Thus, the sample points (Elementary Sample Unit, ESU) all represent an already prepared seedbed pattern, which is the main conditions of fields at this time in the region. As soil surface roughness can be considered anisotropic [3], roughness should be measured in the perpendicular and parallel directions to the seedbed rows. As [4,8] showed a significant impact on the backscattered signal from the periodical roughness component, we focused in this study only on measurements perpendicular to the row directions which is mainly influenced by the periodical component. Table 5 summarizes the main characteristics of each sample point.

\subsection{Geostatistical Analysis}

For the characterization of surface roughness, geostatistical methods were chosen to describe the spatial properties of the soil surface. As variography describes the spatial structure of random spatial objects, it is well suited for the characterization of spatial processes such as soil surface roughness. The omnidirectional sample variogram $\hat{\gamma}(h)$ of a data set can be expressed by:

$$
\hat{\gamma}(h)=\frac{1}{2 n} \sum_{i=1}^{n}\left[Z\left(x_{i}\right)-Z\left(x_{i}+h\right)\right]^{2}
$$


Table 5. Characteristics of sample points acquired within this study, ESU = Elementary Sample Unit.

\begin{tabular}{llll}
\hline ESU & Landuse & Surface Type & Size $\left[\mathrm{m}^{2}\right]$ \\
\hline R11 & maize & seedbed & 6 \\
R12 & onion & smooth crusted & 6 \\
R13 & sugarbeet & crusted seedbed & 8 \\
R14 & sugarbeet & crusted seedbed & 8 \\
R21 & sugarbeet & crusted seedbed & 6 \\
R22 & sugarbeet & crusted seedbed & 6 \\
R23 & sugarbeet & crusted seedbed & 6 \\
R30 & winter rape & seedbed & 22 \\
\hline
\end{tabular}

Figure 3. Overview of roughness sample point on a sugar beet field, acquired on 23 May 2011 at the Wallerfing test site.
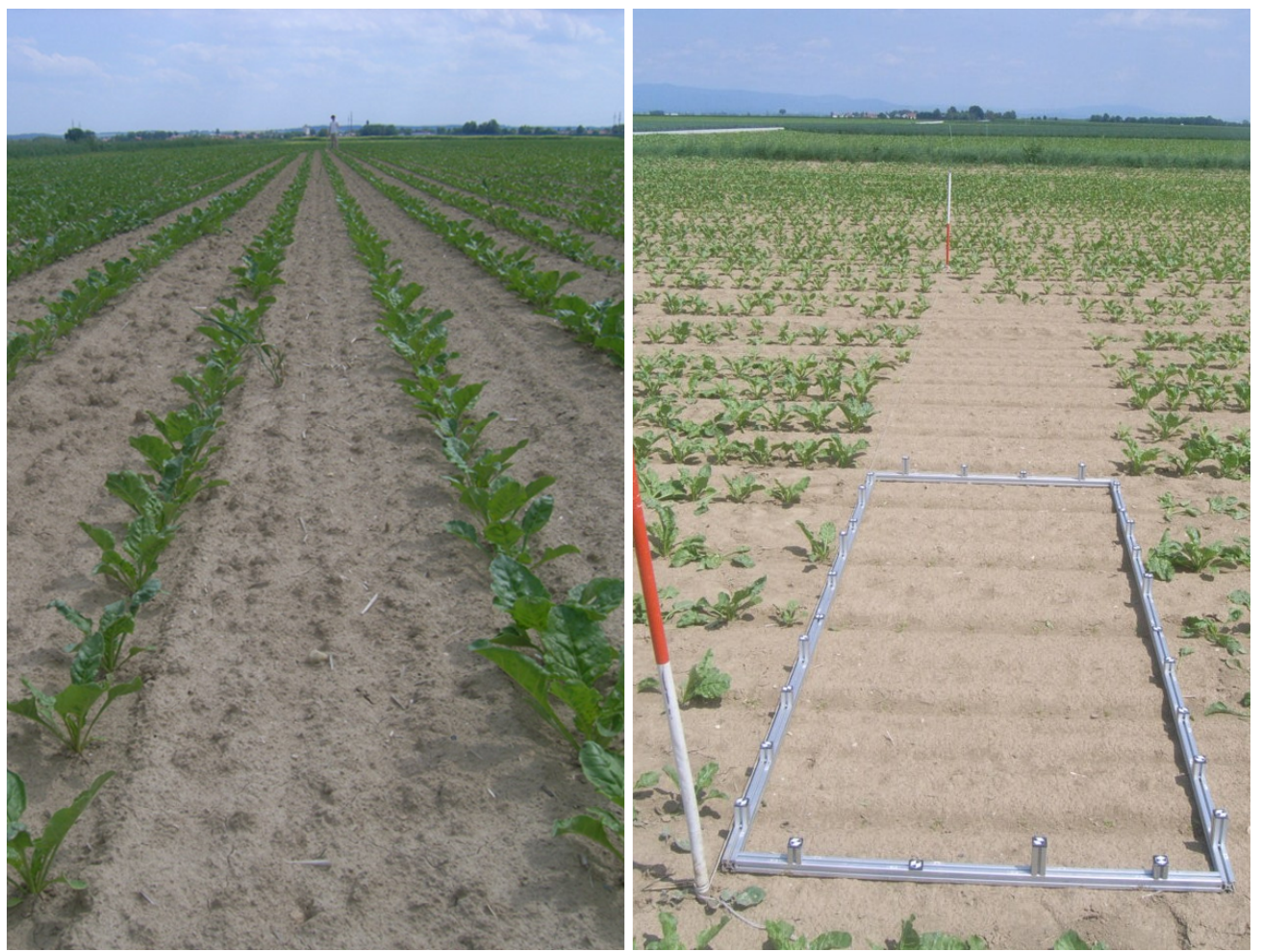

where $h$ is the distance between the two variables $Z\left(x_{i}\right)$ [28]. As the sample design in our study introduces strong anisotropy, one can calculate the sample variogram for a defined direction as

$$
\hat{\gamma}\left(h_{\vec{j}}\right)=\frac{1}{2 n} \sum_{i=1}^{n}\left[Z\left(x_{i}\right)-Z\left(x_{i}+h_{\vec{j}}\right)\right]^{2}
$$

where $\vec{j}$ is the anisotropic direction, in our case $90^{\circ}$. As an example, Figure 4 shows three sample DSMs while Figure 5 shows the corresponding sample variograms of the three samples, calculated on a basis of 15,000 randomly sampled points out of the DSMs. The inherent appearance of the sample variograms displays two major issues: first the surface in Figure 4(b) shows a clear trend, which has to be corrected; 
second, the DSMs perpendicular to the row direction show a two scale process (Figure 4(a)) which has to be quantified for microwave remote sensing separately.

Figure 4. Three sample DSMs of different roughness plots showing (a) a significant two scale roughness pattern (R12), (b) a spatial trend (R14) and (c) no spatial trend with an insignificant two scale roughness pattern (R21). Units are in $\mathrm{cm}$.
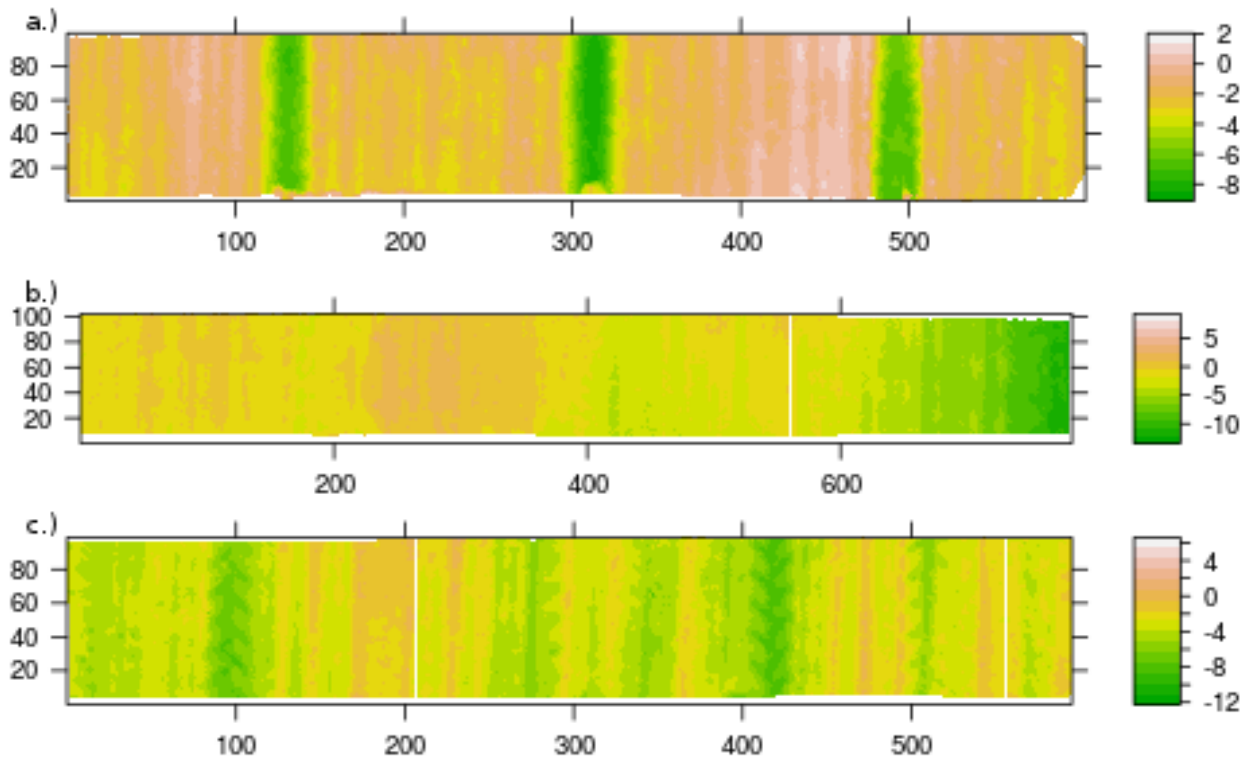

Figure 5. Sample variograms of the roughness plots from Figure 4 showing (a) a significant two scale roughness pattern (R12), (b) a spatial trend (R14) and (c) no spatial trend with an insignificant two scale roughness pattern (R21).
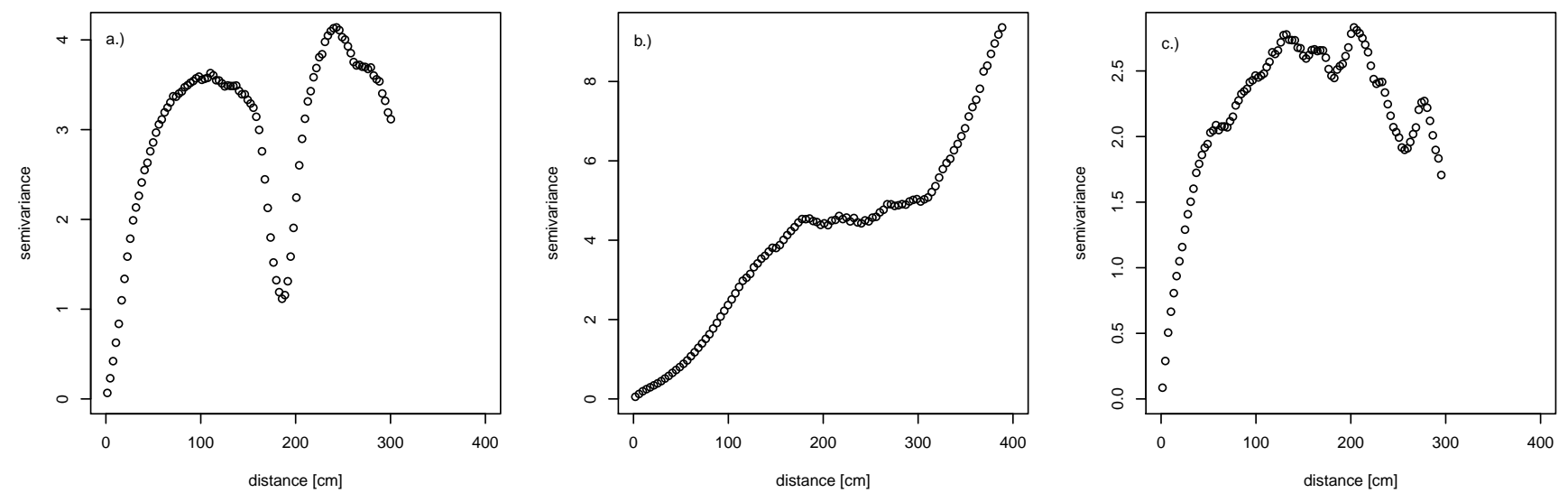

Detrending

As obvious from Figure 4(b), several DSMs showed a trend in elevation due to higher order topographic patterns, such as local slopes. Those slope effects have to be corrected by detrending the data sets. Due to the acquisition design we assume that a spatial trend is only present in the x-direction, while a possible trend in the shorter y-direction $(1 \mathrm{~m})$ can be neglected. Therefore the data can be treated 
as non-spatial data and the detrending can be carried out by subtracting a trend surface $\left(Z_{m o d}\right)$ from the original surface $(Z)$. The detrended surface $\left(Z_{\text {res }}\right)$ is defined as:

$$
Z_{\text {res }}=Z-Z_{\text {mod }}
$$

As summarized in 2nd table in Section 3, two detrending models have been defined, which can be described by

$$
Z_{\text {mod }} \sim m X+b
$$

for the linear model and for the polynomial model:

$$
Z_{\text {mod }} \sim b+m_{1} X+m_{2} X^{2}+m_{3} X^{3}+\ldots m_{n} X^{n}
$$

with $m$ and $b$ representing the regression coefficients slope and intercept and $\mathrm{X}$ the $\mathrm{X}$-coordinate of the sample DSM. Figure 6 shows as an example the results of the detrending procedure, with Figure 6(a) the acquired original surface with a present trend in the right part of the DSM. For detrending a surface is fitted (Figure 6(b)) using a fifth order polynomial approach and subtracted from the original surface to represent a residual surface with a removed trend (Figure 6(c)). As can be seen, the row and seedbed structure is preserved using this approach, allowing the random and orientated roughness components to be quantified. Fitting the trend surface $\left(Z_{\text {mod }}\right)$ to the original heights $(Z)$ by omitting the wheel tracks, a least squares approach using the best fit $\left(\mathrm{R}^{2}\right)$ was chosen.

Figure 6. Results of detrending sample plot R13 with (a) original surface, (b) fitted trend surface using polynomial approach and (c) residual surface preserving row structure. Units in $\mathrm{cm}$.
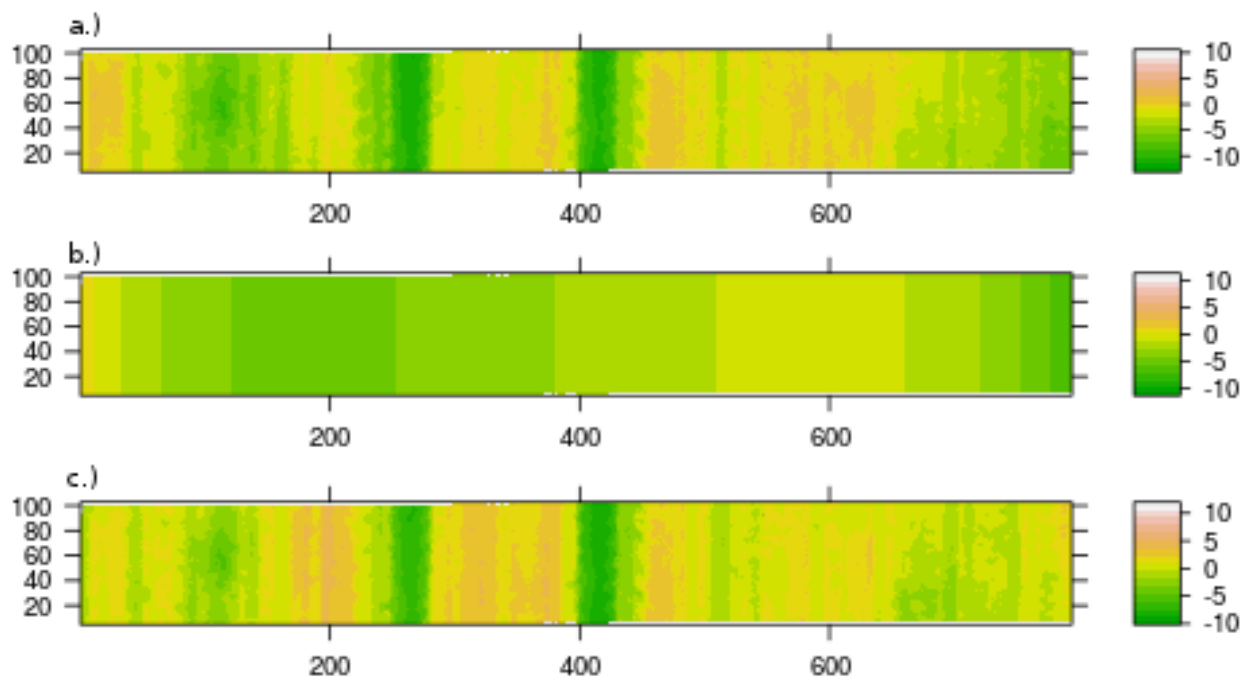

Decomposing the Data into Two Scales

From Figure 4(a) and its corresponding sample variogram (Figure 5(a)) it is obvious that a two scale roughness pattern can be observed on these agriculturally formed soil surfaces. As different roughness scales have an impact on the backscattered signal in microwave remote sensing, it is important to 
characterize both scales. Therefore, again variography is used to decompose and characterize the soil surface at different scales. As the variogram of Figure 5(a) shows a surface inherent behaviour with strong similarities at distances in range of $180 \mathrm{~cm}$, which correspond to the wheel tracks in Figure 4(a), a two scale roughness pattern is indicated. Thus, the variogram for the whole sample plot describes the semi-variance of the roughness pattern, which is strongly imposed by the large scale roughness pattern (e.g., wheel tracks of drilling machine). To characterize the small scale roughness pattern (e.g., seedbed rows, soil clod distribution) we defined a distance threshold, based on the findings of the variography, to mask out the wheel tracks and calculated variograms for each surface again. As a result, for each roughness scale a roughness index is calculated (see Figure 7).

\subsection{Calculation of Roughness Indices}

In order to describe soil surface conditions numerically for remote sensing applications, roughness indices are necessary. In this study, the focus is on the application of the RMS height $s$ and the autocorrelation length $l$, since both are roughness indices used by default in radar remote sensing applications for the characterization of soil surface roughness [1,16,29]. While the RMS height describes the vertical roughness component as the standard deviation of the heights $(Z)$ to a reference height $(\bar{Z})$,

$$
s=\sqrt{\frac{\sum_{i=1}^{n}\left(Z_{i}-\bar{Z}\right)^{2}}{n-1}}
$$

the autocorrelation length $l$ describes the horizontal component of the roughness spectra. While $l$ is usually determined along $1 \mathrm{~d}$ profiles, the derivation of $l$ using three dimensional roughness measurements is more complex. For an efficient estimation of $l$, the autocorrelation function (ACF) can be inverted from a sample variogram $(\hat{\gamma})$ (see Equation (2)), where $l$ is defined as the distance $(h)$ at which the ACF drops under $e^{-1}$ [2]. This implies an exponential fit of the theoretical variogram and therefore of the ACF. Several models were tested, however best fit was always achieved with an exponential model (data not shown). Blaes et al. [2] and Davidson et al. [17] reported similar observations. A theoretical variogram $(\tilde{\gamma})$ with an exponential shape is fitted to the sample variogram $(\hat{\gamma})$ of Equation (2) and from the theoretical variogram $(\tilde{\gamma})$ the $\operatorname{ACF}(\tilde{\rho})$ can be derived as follows:

$$
\tilde{\rho}(h)=1-\frac{\tilde{\gamma}(h)}{\tilde{\gamma}(\infty)}
$$

where $\tilde{\gamma}(h)$ is the modelled semi-variance at distance $h$ between two points and $\tilde{\gamma}(\infty)$ is the modelled semi-variance at distance where the sill of the variogram is reached. For the assumed exponential model, where the sill is asymptomatically approached, $\tilde{\gamma}(\infty)$ corresponds to the distance where $95 \%$ of the sill is reached.

Due to the high resolution of the DSMs, $l$ was calculated using only a random subsample of 15,000 points, while $s$ was calculated for the whole DSM. Therefore, four roughness representations are given for each sample plot (see 2nd table in Section 3). 


\section{Results}

Table 6 shows the root mean square error in the Z-direction $\left(\mathrm{RMSE}_{\mathrm{Z}}\right)$ and the planimetric error of the sample plots. The results show a high accuracy of the generated DSMs compared to the manually measured checkpoints installed on the reference frame, thus providing a robust basis for the characterization of soil surface roughness statistics. Except sample plot R30, which has a RMSE $E_{Z}$ of $0.22 \mathrm{~cm}$. From Figure 4 one can clearly identify the seedbed pattern as well as the nearly random distribution of soil clods and aggregates. The wheel tracks of the different tillage machines used are clearly distinguishable. There are no interpolated artifacts visible which indicate a high rate of matched points during photogrammetric image processing.

Thus, due to the highly accurate results of the DSMs, the calculated roughness indices represent the roughness conditions precisely. While for rougher surface the RMS height $s$ increases, the autocorrelation length $l$ decreases (see Table 7). In general the considered surfaces appear smooth, except for sample plots R11 and R30 which represent a freshly prepared seedbed and therefore show the highest values for $s$ and the lowest values for $l$.

Several studies (e.g., [29]) showed the impact of the sample size on the retrieved roughness values. For the $22 \mathrm{~m}^{2}$ sample plot R30 we analyzed the dependency of the roughness values in context of the sample size according to [7] for the original and the detrended surface. While for the detrended surface the measurements can be assumed to be representative at a sample size of $1.5 \mathrm{~m}^{2}$, the original surface of R30 shows a strong dependency of the roughness values from the sample size which reflects the topographic impact.

Table 6. Root mean square error in $\mathrm{cm}$ of generated DSM height values compared to the manually measured GCPs.

\begin{tabular}{lllllllll}
\hline ESU & R11 & R12 & R13 & R14 & R21 & R22 & R23 & R30 \\
\hline RMSE $_{Z}$ & 0.17 & 0.13 & 0.05 & 0.07 & 0.05 & 0.06 & 0.02 & 0.22 \\
RMSE $_{X Y}$ & 0.03 & 0.04 & 0.04 & 0.04 & 0.11 & 0.04 & 0.04 & 0.04 \\
\hline
\end{tabular}

Table 7. Results of two scale roughness representation and order of fitted detrending surface. In brackets are the results for the non-detrended original surfaces. Units in $\mathrm{cm}, s_{1}, l_{1}$ correspond to the small scale roughness pattern, $s_{2}, l_{2}$ to the large scale roughness pattern.

\begin{tabular}{llllll}
\hline ESU & $s_{1}$ & $s_{2}$ & $l_{1}$ & $l_{2}$ & Detrending \\
\hline R11 & 0.88 & 1.84 & 11.0 & 29.5 & - \\
R12 & 0.85 & 1.73 & 71.6 & 38.27 & - \\
R13 & $1.15(1.09)$ & $2.43(2.84)$ & $38.3(17.7)$ & $53.04(69.05)$ & polynomial 5th order \\
R14 & $0.24(0.86)$ & $2.26(2.5)$ & $41.01(96.39)$ & $98.04(169.14)$ & polynomial 2nd order \\
R21 & 1,24 & 1.45 & 31.1 & 38.52 & - \\
R22 & $0.77(0.93)$ & $1.12(1.51)$ & $20.5(26.4)$ & $23.47(55.4)$ & polynomial 5th order \\
R23 & $1.08(1.31)$ & $2.38(2.71)$ & $27.3(25.7)$ & $105.6(107.5)$ & linear fit \\
R30 & $1.18(2.84)$ & $1.19(3.26)$ & $17.2(145.7)$ & $17.2(359.52)$ & polynomial 9th order \\
\hline
\end{tabular}




\subsection{Effect of Detrending}

Considering only the raw non-detrended dataset, the roughness indices of several surfaces (see Table 7, bracketed values) are influenced by higher order topographic effects such as general slope effects. Figure 4(b) and the corresponding variogram (Figure 5(b)) show this higher order trend for sample plot R14. Using the non-detrended DSM, the calculated roughness indices for $s=2.5$ and $l=169.14$ for the whole dataset are two times the calculated roughness values for the detrended dataset. Thus, the large values, especially for $l$, represent the higher order general slope effects. Indeed the detrended sample plot, using a second order polynomial model according to Table 7 , represents the random and orientated roughness component defined by [9]. Figure 6 shows the output of detrending sample plot R13 using a fifth order polynomial model approach. It is worth highlight that the row structure of the seedbed is preserved by the detrending approach permitting to characterize the periodicity of soil surface roughness as well as the random appearance of single soil aggregates. In Table 7 the roughness indices for the original non-detrended surfaces are provided in brackets. While for the RMS height no significant change is observed due to the detrending procedure, the results for the autocorrelation length $l$ change significantly in the order of several decimetre. Except for R30, the $22 \mathrm{~m}^{2}$ large sample plot, a significant change in $s$ can be observed, due to the strong topographic influence with a range in heights of $20 \mathrm{~cm}$. To model this strong trend a polynomial approach of 9 th order was chosen.

\subsection{Two Scale Roughness Representation}

Figure 5(a) indicates a significant two-scale roughness pattern for sample plot R12. Different points with a certain distance in range of $200 \mathrm{~cm}$ to each other show a strong similarity, thus indicating periodicity in the soil surface roughness pattern with a range of $200 \mathrm{~cm}$. From Figure 4(a) it is obvious that this pattern is clearly related to the wheel tracks of the tillage machines used during seedbed preparation. Thus the sharply bounded wheel tracks with a height difference of 4-6 cm to the surrounding seedbed biases the characterization of the roughness indices. Table 7 shows the results of the decomposing approach for each roughness index and each scale separately. While $s_{1}, l_{1}$ correspond to the small scale roughness pattern (soil clods, soil aggregates and seedbed rows), $s_{2}, l_{2}$ relate to the large scale roughness pattern such as wheel tracks. As the soil surface appeared smooth with only a reduced fraction of the random roughness component (soil clods and aggregates were almost washed out by rain), we decided to comprise the random appearance of soil clods with the seed bed rows in one class separate from the wheel tracks. All sample plots show a two scale roughness representation, due to the availability of a seedbed structure imposed over wheel tracks, at which for the small scale roughness pattern the values for $s_{1}$ and $l_{l}$ are lower than for the large scale roughness pattern. Figure 7 shows the autocorrelation length for three different sample plots. For sample plot R12 (Figure 7(a)), which represents a smooth crusted onion field, the autocorrelation length for the small scale roughness pattern is higher than the large scale roughness pattern, indicating a very smooth surface with sharply bounded wheel tracks (Figure 4(a)). It should be highlighted that even under the same land use type (e.g., sugar beet) the roughness values indicate different roughness conditions, which are a result of the different tillage machines used and the state of crusting. Figure $7(a, b)$ illustrates this effect for sample plots R14 and R21 which both represent sugar beet fields at the same crusted stage. In contrast, sample 
plot R30 shows no significant two scale roughness process, which is due to the missing presence of wheel tracks or other higher order roughness patterns and thus roughness is only defined by the present seedbed structure. As a result, the values of $s$ and $l$ are equal for both scales.

Figure 7. Decomposed two scale roughness pattern for sample plots R12 (a), R14 (b) and R21 (c). Filled dots show the sample variogram and corresponding fitted theoretical variogram (black solid line). Derived autocorrelation function (ACF) $\left(l_{1}\right.$ fine dashed line; $l_{2}$ coarse dashed line) and corresponding autocorrelation length are indicated by hollow dots.
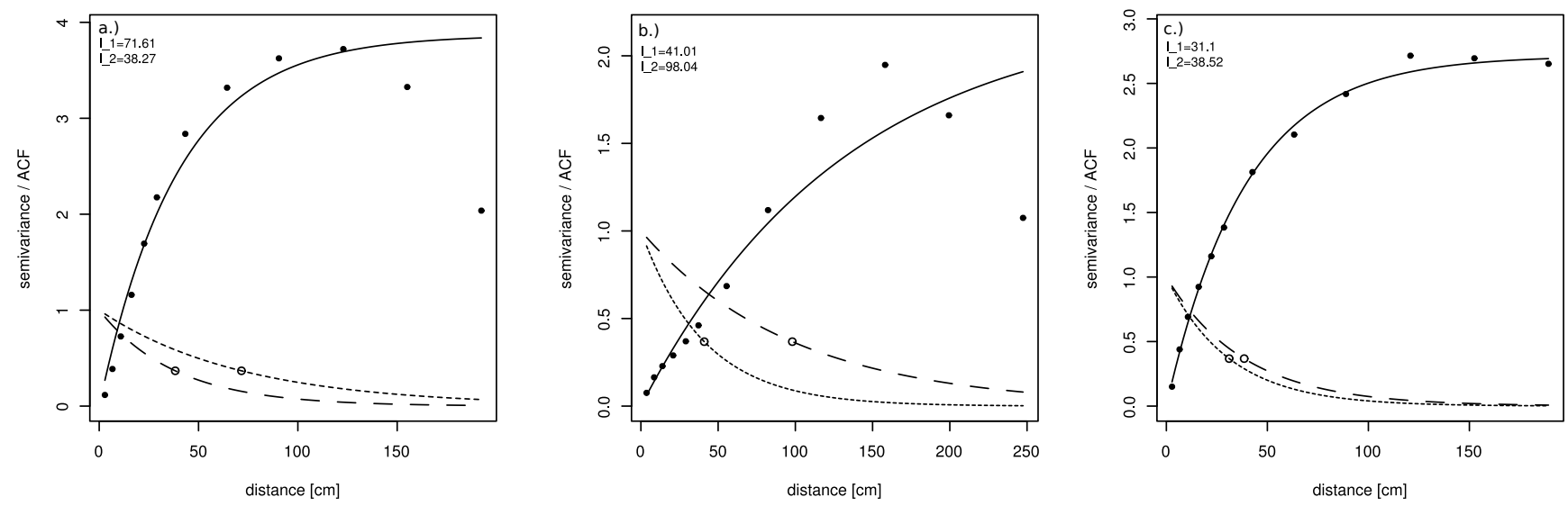

\section{Discussion}

In this paper we presented an approach to characterize the different scales of soil surface roughness for microwave remote sensing applications. Therefore, we established a method based on photogrammetric roughness acquisitions to decompose roughness into two different scales. While the small scale roughness pattern could be clearly related to the seedbed rows of an agricultural field, the large scale roughness pattern is related in to the occurrence of wheel tracks. Both scales are only dependent on the tillage tools used and on the field conditions during tillage operations.

As in microwave remote sensing, soil surface roughness is treated and parametrized in available backscatter models as a single scale stationary process; this approach enables to describe soil surface roughness in such models more accurate [10]. Shin et al. [6,8,30] provide several modelling approaches comprising the roughness scales investigated in this paper. Looking closely to the theoretical study of [8] and comprising the retrieved roughness information into the Modified Shin and Kong Model (MSK) proposed by [8], the findings are in good accordance with our investigations as Figure 8 indicates. Based on our roughness measurements, we used the MSK model to predict the SAR backscattering of several nearly simultaneous acquired ERS-2 datasets. Therefore, we used the derived s1/11 as the random component of the anisotropic roughness and s2/12 as the deterministic sinusoidal component of the MSK model (see [4,8]). As first very preliminary results of this approach show (see Figure 8 and [31]), the results reflect very well the obtained ERS-2 backscatter values and even the directional scattering of the fields with a row orientation nearly perpendicular to the incident wave (off-perp $=0^{\circ}$ ) are modelled accurately. Thus, future studies have to assimilate the output of the proposed method into the available backscatter models of [30] and especially of [8] to verify this first results. 
As shown in this study, both roughness scales differ by several decimetres in the calculated roughness indices, which would thus lead to insufficient results in the inversion of soil moisture by using only a single scale roughness descriptor. While soil surface roughness in an agricultural environment can be considered to be multi-scale, the derived roughness indices are biased by several roughness components. For microwave remote sensing applications it is the two characterized components that have a strong impact on the backscattered signal. Several other roughness components such as general slope effects affect the backscatter signal as well as the proposed roughness measurements. Therefore, the generated DSMs have to be detrended to calculate a roughness index for these two roughness scales. In this study it was obvious that for a surface with medium slope effects, a detrending has a higher impact on the autocorrelation length compared to the RMS height. For surfaces with a keen slope effect, even the results for the RMS height vary strongly.

Figure 8. Backscattering $[\mathrm{dB}]$ versus row direction $\left(0^{\circ}=\right.$ rows perpendicular to the incidence wave) for the roughness samples plots and results of the Modified Shin and Kong model ([8]) averaged for sugar beet parametrization and five ERS-2 scenes (dashed line) acquired nearly simultaneous to the roughness measurements.

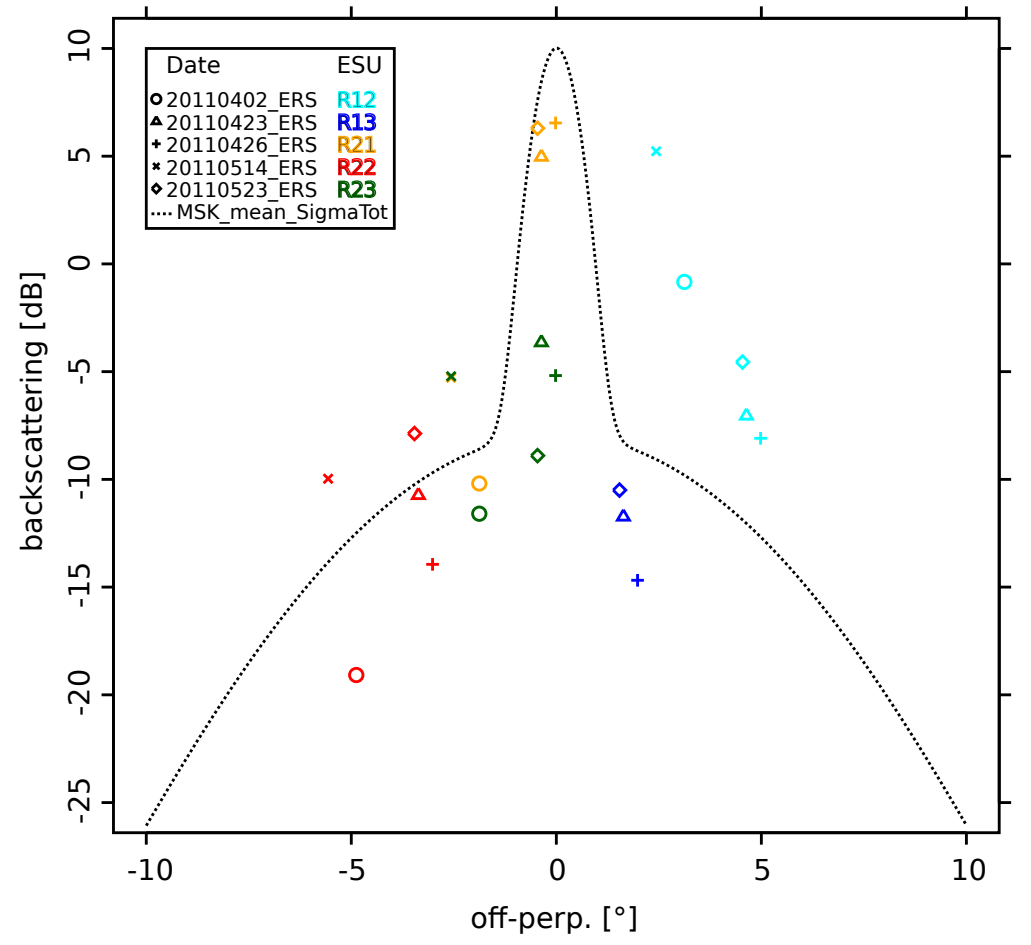

As the findings of this paper are based on highly accurate DSMs, the results are very promising for the parametrization of microwave backscatter models to characterize surface soil moisture. The generated DSMs show a mean RMSE of $0.1 \mathrm{~mm}$ in the Z-direction with a maximum $\mathrm{RMSE}_{\mathrm{Z}}$ of $2.2 \mathrm{~mm}$ and a negligible planimetric error in the sub-pixel region of the imagery. Lievens et al. [32] suggested a threshold for the vertical derivation of heights of $0.2 \mathrm{~cm}$ at which an impact on soil moisture retrieval from microwave imagery is negligible. Thus the proposed roughness acquisition method is well suited for soil surface roughness measurements for microwave remote sensing applications. Using such a setup, no systematic errors in the generated DSMs could be identified, thus the utilization of the customized 
non-metric Canon EOS 5d camera shows a high potential for the measurement of soil surface roughness at reduced costs compared to a metric camera. In the context of field capability, the acquisition time to cover the reference frame is about $15 \mathrm{~min}$, thus outperforming laser devices which need for the same size at same accuracy $2-4 \mathrm{~h}[14,22]$. In addition, as the frame is very portable several roughness measurements can be carried out within a day over several fields.

\section{Conclusions}

In this paper we presented a unique method for measuring soil surface roughness using a simple photogrammetric acquisition system. A consumer grade Canon EOS 5d was customized by fixing the outer lens tube to increase the accuracy of the interior orientation to fulfill metric needs. In combination with a portable reference frame the developed system produces highly accurate digital surface models in any desired size at low cost. Using the system, several roughness measurements were acquired during different field campaigns to characterize soil surface roughness for microwave remote sensing applications. With a vertical accuracy of $\leq 2.0 \mathrm{~mm}$ and a planimetric error smaller than $0.57 \mathrm{~mm}$, the acquired dataset is highly accurate and unique for the characterization of soil surface roughness. Using geostatistical analysis, several roughness scales could be identified, which were decomposed into a large scale roughness pattern (wheel tracks) and a small scale roughness pattern comprising tillage rows and the random appearance of soil clods. Both roughness scales and values were used for the prediction of backscattering using the Modified Shin and Kong model [8] to justify the decomposition of the roughness scales and show its impact on the obtained backscatter values acquired by a SAR sensor. Thus, major progress was achieved in understanding and quantifying the different roughness scales obtained in an agricultural environment leading to a multi-scale roughness parametrization which is mandatory for microwave remote sensing applications.

\section{References}

1. Verhoest, N.; Lievens, H.; Wagner, W.; Alvarez-Mozos, J.; Moran, M.; Mattia, F. On the soil roughness parameterization problem in soil moisture retrieval of bare surfaces from Synthetic Aperture Radar. Sensors 2008, 8, 4213-4248.

2. Blaes, X.; Defourny, P. Characterizing bidimensional roughness of agricultural soil surfaces for SAR modeling. IEEE Trans. Geosci. Remote Sens. 2008, 46, 4050-4061.

3. Marzahn, P.; Ludwig, R. On the derivation of soil surface roughness from multi parametric PolSAR data and its potential for hydrological modeling. Hydrol. Earth Syst. Sci. 2009, 13, 381-394.

4. Wegmuller, U.; Santoro, M.; Mattia, F.; Balenzano, A.; Satalino, G.; Marzahn, P.; Ludwig, R.; Floury, N. Progress in the understanding of narrow directional microwave scattering of agricultural fields. Remote Sens. Environ. 2011, 115, 2423-2433.

5. Ulaby, F.T.; Kouyate, F.; Fung, A.K.; Sieber, A.J. A backscatter model for a randomly perturbed periodic surface. IEEE Trans. Geosci. Remote Sens. 1982, 20, 518-528.

6. Shin, R.T.; Kong, J.A. Scattering of electromagnetic waves from a randomly perturbed quasiperiodic surface. J. Appl. Phys. 1984, 56, 10-21. 
7. Marzahn, P.; Rieke-Zapp, D.H.; Ludwig, R. Assessment of soil surface roughness statistics for microwave remote sensing applications using a simple photogrammetric acquisition system. ISPRS J. Photogramm. 2012, accepted.

8. Mattia, F. Coherent and incoherent scattering from anisotropic tilled soil surfaces. Wave. Random Complex 2011, 21, 278-300.

9. Roemkens, M.J.; Wang, J.Y. Effect of tillage on surface roughness. Trans. ASAE 1986, 29, 429-433.

10. Callens, M.; Verhoest, N.E.C.; Davidson, M.W.J. Parameterization of tillage-induced single-scale soil roughness from 4-m profiles. IEEE Trans. Geosci. Remote Sens. 2006, 44, 878-888.

11. Davidson, M.; Le Toan, T.; Borgeaud, M.; Manninen, T. Measuring the Roughness Characteristics of Natural Surfaces at Pixel Scales: Moving from 1 Metre to 25 Metre Profiles. In Proceedings of 1998 IEEE International Geoscience and Remote Sensing Symposium, Seattle, WA, USA, 6-10 July 1998; Vol. 3, pp. 1200-1202.

12. Alvarez-Mozos, J.; Verhoest, N.; Larranaga, A.; Casalí J.; Gonzáez-Audíana, M. Influence of surface roughness spatial variability and temporal dynamics on the retrieval of soil moisture from SAR observations. Sensors 2009, 9, 463-489.

13. Warner, W.S. Mapping a three-dimensional soil surface with hand-held $35 \mathrm{~mm}$ photography. Soil Till. Res. 1995, 34, 187-197.

14. Aguilar, M.; Aguilar, F.; Negreiros, J. Off-the-shelf laser scanning and close-range digital photogrammetry for measuring agricultural soils microrelief. Biosyst. Eng. 2009, 103, 504-517.

15. Taconet, O.; Ciarletti, V. Estimating soil roughness indices on a ridge-and-furrow surface using stereo photogrammetry. Soil Till. Res. 2007, 93, 64-76.

16. Zribi, M.; Ciarletti, V.; Taconet, O.; Paillé, J.; Boissard, P. Characterisation of the soil structure and microwave backscattering based on numerical three-dimensional surface representation: Analysis with a fractional brownian model. Remote Sens. Environ. 2000, 72, 159-169.

17. Davidson, M.W.J.; Toan, T.L.; Mattia, F.; Satalino, C.; Manninen, T.; Borgeaud, M. On the characterization of agricultural soil roughness for radar remote sensing studies. IEEE Trans. Geosci. Remote Sens. 2000, 38, 630-640.

18. Mattia, F.; Davidson, M.W.J.; Le Toan, T.; D’Haese, C.M.F.; Verhoest, N.E.C.; Gatti, A.M.; Borgeaud, M. A comparison between soil roughness statistics used in surface scattering models derived from mechanical and laser profilers. IEEE Trans. Geosci. Remote Sens. 2003, $41,1659-1671$.

19. Rieke-Zapp, D.; Tecklenburg, W.; Peipe, J.; Hastedt, H.; Haig, C. Evaluation of the geometric stability and the accuracy potential of digital cameras-Comparing mechanical stabilisation versus parameterisation. ISPRS J. Photogramm. 2009, 64, 248-258.

20. Rieke-Zapp, D.H. A digital medium-format camera for metric applications-Alpa 12 metric. Photogramm. Rec. 2010, 25, 283-298.

21. Chandler, J.H.; Fryer, J.G.; Jack, A. Metric capabilities of low-cost digital cameras for close range surface measurement. Photogramm. Rec. 2005, 20, 12-26.

22. Rieke-Zapp, D.; Nearing, M. Digital close range photogrammetry for measurement of soil erosion. Photogramm. Rec. 2005, 20, 69-87. 
23. Aicon 3D Systems. Aicon 3D Studio-User Manual; Aicon 3D Systems: Braunschweig, Germany, 2009; [CD-ROM].

24. Ebner, H. Self Calibrating Block Adjustment. In Proceedings of The XIIIth Congress of the International Society for Photogrammetry, Helsinki, Finnland, 11-23 July 1976; pp. 1-17.

25. Linder, W. Digital Photogrammetry A Practical Course; Springer: Berlin/Heidelberg, Germany, 2009.

26. Heng, P.B.C.; Chandler, .J.H.; Armstrong, A. Applying close range digital photogrammetry in soil erosion studies. Photogramm. Rec. 2010, 25, 240-265.

27. Schlenz, F.; Dall'Amico, J.T.; Loew, A.; Mauser, W. SMOS Validation in the Upper Danube Catchment (UDC): A Status Report Eigth Month after Launch. In Proceedings of ESA Living Planet Symposium 2010, Bergen, Norway, 28 June-2 July 2010.

28. Webster, R.; Oliver, M.A. Geostatistics for Environmental Scientists, 2nd ed.; John Wiley and Sons, LTD: Chichester, UK, 2007; p. 315.

29. Davidson, M.W.J.; Mattia, F.; Satalino, G.; Verhoest, N.E.C.; Le Toan, T.; Borgeaud, M.; Louis, J.M.B.; Attema, E. Joint statistical properties of RMS height and correlation length derived from multisite 1-m roughness measurements. IEEE Trans. Geosci. Remote Sens. 2003, $41,1651-1658$.

30. Zribi, M.; Taconet, O.; Ciarletti, V.; Vidal-Madjar, D. Effect of row structures on radar microwave measurements over soil surface. Int. J. Remote Sens. 2002, 23, 5211-5224.

31. Marzahn, P.; Wegmueller, U.; Mattia, F.; Ludwig, R. FLASHING FIELDS! and the Impact of Soil Surface Roughness. In Proceedings of IEEE International Geoscience and Remote Sensing Symposium IGARSS 2012, Munich, Germany, 22-27 July 2012.

32. Lievens, H.; Vernieuwe, H.; Alvarez-Mozos, J.; De Baets, B.; Verhoest, N. Error in radar-derived soil moisture due to roughness parameterization: An analysis based on synthetical surface profiles. Sensors 2009, 9, 1067-1093.

(C) 2012 by the authors; licensee MDPI, Basel, Switzerland. This article is an open access article distributed under the terms and conditions of the Creative Commons Attribution License (http://creativecommons.org/licenses/by/3.0/.) 